\title{
Review of Lawrence A. Boland's Model building in economics: its purposes and limitations. Cambridge: Cambridge University Press, 2014, 298 pp.
}

\author{
JAAKKO KUORIKOSKI \\ University of Helsinki
}

The purposes and limitations of all types of models used in economics, past and beyond. This is the rather ambitious topic of Lawrence Boland's new book. The book reviews standard microeconomics, macroeconomics, game theory (including evolutionary), experimental economics, and econometrics (both micro and macro). In its mission statement, the book is declared methodology with a small ' $m$ ', to be distinguished with a capitalized Methodology discussed by Philosophers. Methodology discussed by Philosophers deals with lofty issues of 'Realism', 'Testing', and 'Explanation', whereas methodologists are interested in, well, such things as the realisticness of assumptions, the testing of models, and explanation. In the end, the biggest drawback of the book ends up being a seemingly aesthetic aversion to completely imagined capital letters. It is clear that the book discusses the very same issues that have vexed philosophers of science and philosophers of economics for at least the last few decades, and arguably for centuries, yet it refuses to engage these resources. This is a regrettable, but perhaps inevitable, consequence of the limits of any one person trying to cover the whole of economics and the relevant philosophy of economics.

Boland begins with a thesis about a paradigm and generational shift in economic modeling around the beginning of the 1980s. Economists having their postgraduate studies in the 1980s or later no longer conceive models as being models of a theory, built in order to provide an instrument with which to empirically test that theory. Instead, model building began to be equated with theorizing itself. This shift is argued for in a concise, yet convincing manner. And this shift does not sit all that well with the Popperian spirit of Boland's Methodology (yes, with a capital 'M'). Although the book does not attempt to systematically build a single argument or an encompassing philosophical position, the overall message emerging from the discussion seems to be a plea for 
more interest in the realisticness and empirical testing of modeling assumptions.

The book first goes through the stylized history of the division between micro and macroeconomics. It dates the beginning of modern microeconomics to Marshall and proceeds from there to discuss the need for, and the plausible form of, microfoundations. Standard criticisms against the use of representative agent constructs and the sterility of general equilibrium theory are also raised. The biggest faults located by Boland in theoretical macro models based on equilibrium assumptions are their lack of dynamics and any idea of adjustment process for prices. There is nothing new in these accusations, but Boland raises the issues with a peculiar twist. It would not, in fact, be enough to endow the model with dynamics by having the endogenous variables be determined by their lagged values and additional exogenous variables. This would be merely a combination of exogenous shocks and a predetermined time path, not truly endogenous dynamics resulting from the actions of free agents. Approvingly quoting Hicks, Boland holds that economics must not only include time, but be in time.

The argument here is that the problem is not just in the lack of an empirically credible adjustment process, but in the fact that the conceptions of learning and expectation formation implicit in any proposed sketch of an adjustment process are flawed, because they have always been based on inductive reasoning. And inductive reasoning was demonstrated to be impossible by Hume. This foundational problem of the groundlessness of inductive learning is also raised with respect to agents within game-theoretical models: common knowledge of rationality either assumes a foundationally irrational inductive learning process or simply assumes away the whole 'problem of induction'. Even more generally, all economic applications of game and decision theory are suspect to the extent they appeal to probabilities and thus conflate decision under risk and uncertainty.

Now, there is certainly nothing wrong with taking such a strong position with respect to the roles of probability and inductive reasoning. Boland's deep mistrust of both notions is very much in line with his general Popperian outlook: there is no quantitative (probabilistic) measure of (inductive) evidential support and the only respectable use of probability concepts is as propensities of well-defined probability setups (such as games of chance). What is problematic is that these stances are not really argued for, but simply claimed to be the only rationally 
acceptable positions. In this book Boland thus inadvertently manages to show how deep Methodological commitments have direct implications to model choice and testing, but at the same time refuses to acknowledge or really argue for the adopted big-M presuppositions. Inductive reasoning is bogus because of Hume and probabilities should not be trusted because the world is really not stochastic. I fully agree with Boland in that too much of game theory is motivated by purely mathematical and conceptual puzzles (such as the conceptual mire of knowledge and belief, unnecessarily covered at length also in this book) whereas too little attention is spared for methodological reflection on how these models are supposed to connect with economic reality. I am not just sure whether the arguments presented for this claim are the most convincing ones.

And what goes for reasoning within the models also goes for reasoning about models. It comes as no surprise that Boland's Popperian outlook is not hospitable to Bayesian philosophy of science, but it would have been nicer to acknowledge its existence and even review some of its claimed advantages, rather than dismissing the very possibility of such an enterprise as if resting on a simple failure to understand elementary logic or as an illegitimate act of moving of the goal posts after failing to score.

Instead of inductive reasoning, Boland advocates a simple hypothetico-deductive picture of testing. But in contrast to Popper and his followers, he aims to actually overcome the Duhem-Quine (D-Q) thesis and the problem of underdetermination by providing an account of "logically adequate" model testing. Boland first makes an important distinction between testing a substantial theoretical assumption (a behavioral hypothesis) and testing a specification assumption linking the first kind of assumption to empirical data. The way to test a specific behavioral hypothesis in a logically adequate way is to construct a model with an alternative behavioral hypothesis but similar specification assumptions-a counter-example to the original hypothesis-and seeing which fits better. If the counter-example fits but the original does not, then the behavioral hypothesis is refuted. This certainly sounds sensible and fits well with the contrastive ideas about testing and confirmation, as well as with the ideas about testing specific modeling assumptions using robustness analysis and the like, currently vigorously discussed in philosophy of science. Nevertheless, it is obvious that this is not a logically foolproof solution to the D-Q 
"problem". There will still always remain logically possible alternative explanations for why one model fits whereas the other does not. The DQ thesis simply does not admit of such a conclusive "solution".

It is clear that we are now engaged in Methodology with a giant M. And it is a missed opportunity that the book does not discuss other currently viable Popperian views, such as the severe testing framework. This is all the more puzzling since Boland is happy to review (approvingly) the work of Aris Spanos on the importance of statistical misspecification testing, but either ignores or refuses to engage with the broader Methodological picture painted by him in collaboration with Deborah Mayo (see, e.g., essays in Mayo and Spanos 2010). Also much of the discussion on the way that empirical models confront the empirical world could have been much more illuminating if structured along the Bogen-Woodward data-phenomena-theory framework (Bogen and Woodward 1988). Now, the discussion on testing is conceptualized as that of testing theoretically motivated models directly with observations (even token ones!). Treating the data-phenomenon inference as a separate epistemic step would, in my mind, make more sense of much of empirical practice and is, arguably, also a more charitable interpretation of such diverse things as the casual appeal to stylized facts, the role of laboratory experimentation, and the LSE-methodology in macroeconometrics.

When it comes to econometrics, especially macroeconometrics, the book criticizes much of empirical practice as necessarily presupposing that human actions are predetermined by nature-given and immutable parameters. This is not conceived of only as an empirical shortcoming, but as some sort of conceptual folly or a symptom of a world-view antagonistic towards human autonomy. Yet again, the biggest shortcoming in the way the book covers the central methodological disputes around econometric modeling is that it ignores the advances in understanding causality and prediction versus accommodation achieved in philosophy of science in the last few decades. For example, Boland seems to conflate the (completely reasonable) worry that any estimated parameter values may not be stable across time with the question of whether deep parameters invariant under exogenous interventions can be uniquely identified given the available data and restrictions. Yet it is arguably the latter concern that limits the usefulness of completely atheoretical VAR models. And again, there is an opportunity missed in that Boland does not discuss the important work of Kevin Hoover on the 
methodology of macroeconometrics-work (e.g., Hoover 2001; Hoover 2010) that surely should have qualified as small-m methodology given Hoover's disciplinary affiliation.

In sum, the book's breadth of scope is breathtaking and it is a treasure trove of useful references for further pursuing more specific questions. I certainly learned a lot reading this book. But in trying to cover every aspect of orthodox economic practice the book, perhaps inevitably, fails to really develop a comprehensive and coherent methodological view from which to evaluate or perhaps even offer guidance concerning the activities in question. This robs the book much of its reason d'être. It covers a bewildering array of topics, and there are a lot of astute observations about them, but it is not quite clear to what ultimate end. There is certainly a strong methodological view present in the book, but it is left mostly implicit and scantly argued for. The refusal to explicitly engage with issues that are perceived as too philosophical does not mean that methodological reflection could proceed entirely without commitments to such issues-it just means that the resulting overall picture ends up feeling somewhat idiosyncratic and lacking in foundations.

But perhaps the biggest omission arising from snubbing philosophy of science relates to the title: considering that the book is not just about the limitations of models, but also about their purposes, it is peculiarly silent about what is arguably the main purpose of most highly idealized theoretical models, namely explanation or "sense-making". But delving into this territory would probably have meant discussing more Methodology with a capital M.

\section{REFERENCES}

Bogen, James, and James Woodward. 1988. Saving the phenomena. Philosophical Review, 97 (3): 303-352.

Hoover, Kevin. 2001. Causality in macroeconomics. Cambridge: Cambridge University Press.

Hoover, Kevin. 2010. Idealizing reduction: the microfoundations of macroeconomics. Erkenntnis, 73 (3): 329-347.

Mayo, Deborah, and Aris Spanos (eds.). 2010. Error and inference: recent exchanges on experimental reasoning, reliability, and the objectivity and rationality of science. Cambridge: Cambridge University Press

Jaakko Kuorikoski is currently a professor in social and moral philosophy at the University of Helsinki and a research fellow at TINT 
Academy of Finland Centre of Excellence in the Philosophy of the Social Sciences. He has published on explanation, models and simulations, mechanisms, and causality in, e.g., British Journal for the Philosophy of Science, Erkenntnis, Philosophy of Science, Philosophy and Phenomenological Research, and Synthese. His current research interests include evidence, macroeconomic modeling, and the social and policy relevance of neuroscience.

Contact: <http://jaakkokuorikoski.wordpress.com/> 